\title{
DIRECTED PACKINGS OF PAIRS INTO QUADRUPLES
}

\author{
DAVID B. SKILLICORN
}

(Received 10 August 1981)

Communicated by W. D. Wallis

\begin{abstract}
A directed packing of pairs into quadruples is a collection of 4-subsets of a set of cardinality $v$ with the property that each ordered pair of elements appears at most once in a 4-subset (or block). The maximal number of blocks with this property is denoted by $D D(2,4, v)$. Such a directed packing may also be thought of as a packing of transtivie tournaments into the complete directed graph on $v$ points. It is shown that, for all but a finite number of values of $v, D D(2,4, v)$ is maximal.
\end{abstract}

1980 Mathematics subject classification (Amer. Math. Soc.): 05 B 40.

\section{Introduction}

A directed packing is a collection of $k$-subsets (called blocks) of a set of cardinality $v$ with the property that every ordered $t$-subset appears in at most one block. A $t$-set is contained in a $k$-set if its symbols appear in order, possibly interspersed with other symbols. Thus, the block abcd contains the six pairs: $a b, a c, a d, b c, b d$ and $c d$. The maximal number of blocks with this property is denoted by $D D(t, k, v)$ for each choice of $t, k$ and $v$. In this paper, directed packings of pairs into quadruples are considered.

A counting argument can be used to derive an upper bound on the value of $D D(2,4, v)$. As no ordered pair can appear more than once, no symbol can appear in more than $2(v-1)$ pairs. Therefore its frequency cannot be greater than L2(v-1)/3」 since each time it appears in a block it appears in 3 pairs. Summing frequencies over all symbols gives

$$
D D(2,4, v) \leqslant\left\llcorner\frac{v}{4}\left\llcorner\frac{2(v-1)}{3}\right\lrcorner\right\lrcorner .
$$

Call the right-hand side of (1), $U(v)$.

C Copyright Australian Mathematical Society 1982 
A lower bound may be derived by considering ordinary packings. A packing may be made into a directed packing by writing each block of the packing twice, once forward and once reversed. This gives the lower bound

$$
D D(2,4, v) \geqslant 2 D(2,4, v) \text {. }
$$

\section{The easy cases}

D. J. Street and J. R. Seberry [4] have shown that, when $v \equiv 1(\bmod 3)$, a directed packing exists which contains every ordered pair exactly once. Such a structure is called a directed balanced incomplete block design and is of special interest having, for example, some of the statistical sampling properties of row complete latin squares. So we have

THEOREM 1. If $v \equiv 1(\bmod 3)$ then

$$
D D(2,4, v)=U(v) .
$$

When $v \equiv 2(\bmod 3)$, A. E. Brouwer [1] has shown that

$$
D(2,4, v)=\left\llcorner\frac{v}{4}\left\llcorner\frac{v-1}{3}\right\rfloor\right\lrcorner .
$$

This result can be used to prove

THEOREM 2. If $v \equiv 2(\bmod 3)$ then

$$
D D(2,4, v)=U(v) \text {. }
$$

ProOF. (1) and (2) give, when $v \equiv 2(\bmod 3)$

$$
2\left\llcorner\frac{v}{4}\left\llcorner\frac{v-1}{3}\right\lrcorner\right\lrcorner \leqslant D D(2,4, v) \leqslant\left\llcorner\frac{v}{4}\left\llcorner\frac{2(v-1)}{3}\right\lrcorner\right\lrcorner .
$$

The left- and right-hand sides are equal, implying the result.

\section{An indirect product construction}

THEOREM 3. If there are directed packings on $w$ and $v$ points such that $D D(2,4, w)=n$ and $D D(2,4, v)=m$ then

$$
D D(2,4, w(v-b)+b) \geqslant w m+n(v-b)^{2}
$$

for all $b=0,1$ such that $v-b \in O A(4)$ (that is $v-b \neq 2$ or 6 for which there do not exist 2 mutually orthogonal latin squares). 
Proof. Take $w$ sets of size $v$ which are disjoint except for $b$ points which are common to all of them. Call these sets $C_{i}(i=1,2, \ldots, w)$. From $w$ copies of the packing on $v$ points on these sets (since $b \leqslant 1$ there are no repeated pairs). The pairs from distinct sets are included as follows: take $n$ orthogonal arrys of size 4 by $(v-b)^{2}$ and index the columns so that if $p_{1} p_{2} \cdots p_{4}$ is a block of the packing on $w$ points then one of the orthogonal arrays has rows of the form $x_{p_{1}} x_{p_{2}} \cdots x_{p_{4}}$.

All ordered pairs from within each of the $C_{i}$ s may only appear in the packing written on $C_{i}$. Ordered pairs of the form $x_{i} y_{j}(i \neq j)$ may only appear in the orthogonal array indexed by the block containing $i j$. The constructed object is therefore a directed packing on $w(v-b)+b$ points and has $w m+n(v-b)^{2}$ blocks.

This theorem is useful when the lower bound on the right-hand side equals the upper bound (1). This happens in the following cases.

CoRollary 4. If $w \equiv 1(\bmod 3)$ and $v \equiv 0(\bmod 12)$ and $D D(2,4, v)=U(v)$ then

$$
D D(2,4, w v)=U(w v)
$$

Corollary 5. If $w=4,7$ or 10 and $v \equiv 0(\bmod 12)$ and $D D(2,4, v)=U(v)$ then

$$
D D(2,4, w(v-1)+1)=U(w(v-1)+1) .
$$

To use these results, maximal packings with $v$ in the congruence class $0(\bmod 12)$ must be constructed. The two smallest cases are shown below.

$D D(2,4,12)=21$.

$$
\begin{aligned}
& 1_{1} 0_{2} 1_{2} 0_{1} \\
& \mathrm{O}_{4} \mathrm{2}_{4} \mathrm{O}_{3} \mathrm{O}_{1} \\
& 2_{2} \mathrm{l}_{2} \mathrm{O}_{3} \mathrm{O}_{4} \\
& I_{3} 2_{1} 0_{2} 0_{3} \text { all developed } \bmod 3 \text {. } \\
& 2_{1} 0_{1} 2_{3} 0_{4} \\
& 2_{4} 0_{2} 1_{1} 0_{4} \\
& 2_{3} \mathrm{l}_{4} \mathrm{O}_{3} \mathrm{O}_{2}
\end{aligned}
$$


$D D(2,4,24)=90$.

$$
\begin{array}{llll}
0_{2} 0_{1} 0_{3} 0_{4} & 4_{2} 5_{4} 2_{1} 0_{4} & \\
0_{4} 0_{3} 1_{2} 0_{1} & 3_{4} 5_{2} 1_{3} 0_{4} & \\
0_{1} 1_{4} 2_{3} 0_{2} & 1_{1} 3_{1} 4_{3} 0_{4} & 5_{3} 1_{3} 2_{3} 0_{4} \\
1_{3} 3_{1} 4_{2} 0_{1} & 3_{2} 5_{2} 2_{4} 0_{1} & \text { all developed } \\
0_{3} 4_{3} 1_{1} 0_{2} & 1_{4} 5_{4} 4_{4} 0_{1} & \text { mod } 6 . \\
21_{1} 1_{3} 4_{4} 0_{3} & 3_{1} 4_{1} 2_{1} 0_{2} & \\
1_{4} 3_{4} 1_{2} 0_{3} & 5_{2} 3_{2} 2_{2} 0_{3} &
\end{array}
$$

Construction of the remaining packings in this class depends on the following theorem.

THEOREM 6 [2, Brouwer, Hanani, Schrijver]. Necessary and sufficient conditions for the existence of a group divisible design on voints with blocks of size 4 and groups of size $m$ are that $v \equiv 0(\bmod m), v-m \equiv 0(\bmod 3)$ and $v=m$ or $v \geqslant 4 m$ (except for two cases $v=8, m=2$ and $v=24, m=6$ ).

THEOREM 7. If $\Delta(v)=U(v)-D D(2,4, v)$ then

$$
\Delta(12 v) \leqslant v \Delta(12) \cdot \text { for } v \geqslant 4
$$

Proof. By Theorem 6, there exists a group divisible design on $12 v$ points $(v \geqslant 4)$ with $v$ groups of size 12 and $24 v^{2}-2 v-10$ directed blocks. Replacing each group by a directed packing on 12 points, the result follows.

In fact, since $D D(2,4,12)=21$ it follows that

Theorem 8. If $v \equiv 0(\bmod 12), v \neq 36$, then

$$
D D(2,4, v)=U(v) \text {. }
$$

Theorem 8, together with Corollaries 4 and 5 , give

THEOREM 9. For $v$ in the following congruence classes, $D D(2,4, v)=U(v)$ :

$$
\begin{aligned}
v & \equiv 45(\bmod 48), & v \neq 141, \\
v & \equiv 78(\bmod 84), & v \neq 246, \\
v & \equiv 111(\bmod 120), & v \neq 351 .
\end{aligned}
$$




\section{A recursive construction}

THEOREM 10. If $m \in O A(10), m \equiv 0(\bmod 12), D D(2,4, m)=U(m)$ and $0 \leqslant t$ $\leqslant m$ then

$$
\Delta(10 m+3 t) \leqslant \Delta(m+3 t) .
$$

Proof. The following directed group divisible designs exist:

1. $D G D D$ on 10 points with 10 groups of size 1 and 15 directed blocks.

2. $D G D D$ on 13 points with 1 group of size 4,9 groups of size 1 and 24 directed blocks.

Take a transversal design with 10 groups of size $m$. Use the construction of R. M. Wilson [5, Fundamental Construction] giving each point a weight of 1 except $t$ points in a single group given a weight of 4 . The resulting directed group divisible design has 9 groups of size $m, 1$ group of size $m+3 t$ and $m(15 m+9 t)$ blocks. Replacing each of the groups by a directed packing of an appropriate size results in a directed packing on $10 m+3 t$ points, the number of whose blocks falls short of the upper bound by at most as much as the directed packing on $m+3 t$ points.

THEOREM 11. If $v$ is sufficiently large then

$$
D D(2,4, v)=U(v)
$$

Proof. By Theorem 8, if $v \equiv 0(\bmod 12)$ and $v>36$ then $D D(2,4, v)=U(v)$.

If $v \equiv 3(\bmod 12)$ and $0 \leqslant t \leqslant 399$ then $v$ can be expressed as $10 m+3 t$ in such a way that $m+3 t \equiv 111(\bmod 12)$. The largest $m$ not known to be in $O A(10)$ is $m=3576$ so that if $m>3576$ the conditions of Theorem 10 are satisfied. Therefore if $v \geqslant 35778$ and $v \equiv 3(\bmod 12)$ then $D D(2,4, v)=U(v)$.

If $v \equiv 6(\bmod 12)$ and $0 \leqslant t \leqslant 279$ then $v$ can be expressed as $10 m+3 t$ with $m+3 t \equiv 78(\bmod 84)$. If $v \geqslant 35778$ the result follows.

Similarly if $v \equiv 9(\bmod 12)$ and $0 \leqslant t \leqslant 159$ then $v$ can be written as $10 m+3 t$ in such a way that $m+3 t \equiv 45(\bmod 48)$ and if $v \geqslant 35781$ the result follows.

Using Theorem 3, a computer program was run to determine which other packings are maximal using $m$ values known to be in $O A(10)$. This showed that, in fact, if $v>15579$ then $D D(2,4, v)=U(v)$. A list of values of $v$ for which $D D(2,4, v)$ is not known to be maximal can be found in [3]. 


\section{References}

[1] A. E. Brouwer, 'Optimal packings of $K_{4} s$ into a $K_{n}$ ', $J$. Combinatorial Theory $B 26$ (1979), 278-297.

[2] A. E. Brouwer, H. Hanani and A Schrijver, 'Group divisible designs with block size four', Discrete Math. 20 (1977), 1-10.

[3] D. B. Skillicorn, Directed packings and coverings with computer application (Ph.D. Thesis, University of Manitoba, 1981).

[4] D. J. Street and J. R. Seberry, 'All DBIBDs with block size four exist', Utilitas Math. 17 (1980), 27-34.

[5] R. M. Wilson, 'Construction and uses of pairwise balanced designs', Proc. of NATO Advanced Study Inst. on Combinatorics, Nijenrode Castle, Brukelen (1974), pp. 19-42.

Department of Mathematics, Statistics and Computing Science

Dalhousie University

Halifax, Nova Scotia B3H 4H8

Canada 